\title{
Amyloid formation by short peptides in the presence of dipalmitoylphosphatidylcholine membranes
}

Article

Accepted Version

Gerbelli, B. B. ORCID: https://orcid.org/0000-0001-9300-8463, Oliveira, C. L. P. ORCID: https://orcid.org/0000-0002-34266507, Silva, E. R. ORCID: https://orcid.org/0000-0001-58762276, Hamley, I. W. ORCID: https://orcid.org/0000-0002-45490926 and Alves, W. A. ORCID: https://orcid.org/0000-00028394-2751 (2020) Amyloid formation by short peptides in the presence of dipalmitoylphosphatidylcholine membranes. Langmuir, 36 (48). pp. 14793-14801. ISSN 0743-7463 doi: https://doi.org/10.1021/acs.langmuir.0c02760 Available at https://centaur.reading.ac.uk/94834/

It is advisable to refer to the publisher's version if you intend to cite from the work. See Guidance on citing.

To link to this article DOI: http://dx.doi.org/10.1021/acs.langmuir.0c02760

Publisher: American Chemical Society 
copyright holders. Terms and conditions for use of this material are defined in the End User Agreement.

\section{www.reading.ac.uk/centaur}

\section{CentAUR}

Central Archive at the University of Reading

Reading's research outputs online 


\section{Amyloid Formation by Short Peptides in the Presence of Dipalmitoylphosphatidylcholine Membranes}

Barbara B. Gerbelli, ${ }^{[a]}$ Cristiano L. P. Oliveira, ${ }^{[b]}$ Emerson R. Silva,${ }^{[c]}$ Ian W. Hamley, ${ }^{[\mathrm{d}]}$ Wendel A. Alves ${ }^{[\mathrm{a}], *}$

${ }^{[a]}$ Centro de Ciências Naturais e Humanas, Universidade Federal do ABC, Santo André 09210-580, Brazil.

${ }^{[b]}$ Instituto de Física, Universidade de São Paulo, São Paulo 05314-970, Brazil.

${ }^{[c]}$ Departamento de Biofísica, Universidade Federal de São Paulo, São Paulo 04023-062, Brazil.

${ }^{[d]}$ Departmet of Chemistry, University of Reading, Reading RG6 6AD, United Kingdom.

*Corresponding author: wendel.alves@ufabc.edu.br 


\begin{abstract}
The aggregation of two short peptides $[R F]$ and $[R F]_{4}$ (where $\mathrm{R}=$ arginine and $\mathrm{F}=$ phenylalanine) at dipalmitoylphosphatidylcholine (DPPC) model membranes was investigated at the air-water interface using the Langmuir technique and vesicles in aqueous solutions. The molar ratio of the peptide and lipid components was varied to provide insights into the peptide-membrane interactions, which might be related to their cytotoxicity. ${ }^{1}$ Both peptides exhibited affinity to the DPPC membrane interface and rapidly adopted $\beta$-sheet rich structures upon adsorption onto the surface of the zwitterionic membrane. Results from adsorption isotherm and small angle X-ray scattering (SAXS) experiments showed changes in the structural and thermodynamic parameters of the membrane with increasing peptide concentration. Using atomic force microscopy (AFM), we showed the appearance of pores through the bilayer membranes and peptide aggregation at different interfaces, suggesting that the hydrophobic residues might have an effect on both pore size and layer structure, facilitating the membrane disruption and leading to different cytotoxicity effects.
\end{abstract}

Keywords: Lipid monolayers, Langmuir-Blodgett films, peptide, AFM, SAXS, amyloid.

\title{
1. INTRODUCTION
}

The assembly of amyloidogenic peptides such as Amyloid $\beta$ (A $\beta$ ) with model membranes provides a way to investigate the mechanisms behind the action of oligomers on biomembranes. These assemblies are formed in the close vicinity of the lipid interface, and they are thought to alter the membrane structure and lead to cell lysis and death. Several experiments have contributed to the understanding of how $A \beta$ influences the behaviour of lipid membranes. ${ }^{2-5}$ A significant breakthrough was the demonstration that amyloidogenic peptides can assemble into annular structures, inducing amyloid pores in the membrane with ion channel properties. ${ }^{6,7}$ Membrane defects in the presence of this class of peptides are similar to those induced by antimicrobial peptides for bacterial membrane permeation, suggesting that universal principles govern their folding and functions. ${ }^{8}$ However, these experiments did not reveal the molecular mechanisms involved in the different modes of amyloid oligomerization and aggregation. 
In recent years, model sequences containing important segments of the $A \beta$ peptide have been investigated by several authors using model membranes to study channel formation in planar lipid bilayers, as well as in biological membranes in living cells. 5 , 6, 9, 10 The hydrophobic residues seems to influence the insertion, cytotoxicity, and pore formation of the liposome membrane. ${ }^{11-13}$

Several works have explored the sequence of short model peptides, which form supramolecular $\beta$-sheets and amyloid-like fibrils upon self-association. ${ }^{14-18}$ Notably, we found that the self-assembly of a model octapeptide consisting of an alternating sequence of arginine (R) and phenylalanine (F) residues leads to highly ordered and extended amyloid fibrils, which produce a very high quality "cross- $\beta$ " XRD pattern. ${ }^{15}$ Changes in the morphology of these aggregates were observed as the length of the peptide was increased from $[R F]$ to $[R F]_{5}$, promoting a fibril-forming propensity. In contrast, shorter peptides form fractal-like aggregates. ${ }^{1}$

Our previous experimental studies allow us to compare the effect of charge, independent of the secondary structure. All peptides exhibited cytotoxicity for glioma and RAW264.7 macrophage cells at concentrations comparable to the critical aggregation concentrations ( $c a c$ ) found in water, suggesting that a nonspecific mechanism causes cell death and that cytotoxicity assays are dependent on peptide length. ${ }^{1}$ However, there is a need for simpler model systems that could assist elucidation of the mechanisms involved in peptide-membrane interactions which may underlie their cytotoxic properties.

In this present work, to investigate permeability we use the DPPC model system, which is a lipid suited to mimic cell membranes. Here we have analysed the surface properties and lipid-peptide interactions of two peptides $[R F]$ and $[R F]_{4}$, which are characterized by different solubility and morphology in aqueous solution with DPPC, using both monolayers and vesicles as membrane-substrate interfaces. Lipid monolayers (2D) enable the direct exploration of the local interaction among the lipid layer and biomolecules, for example, accessing the changes in the organization of lipids and the area expansion of monolayers induced by peptide-membrane intercalations. On the other hand, liposomes are attractive models because they offer a three-dimensional (3D) representation of the cell membranes and allow the observation of global changes in the layer promoted by peptides.

The experimental evidence presented in this study allows us to propose a multistage model based on that of Hirst et al., ${ }^{19}$ in which peptide and lipid interactions 
occur in three steps. The first stage describes the strong interaction of the peptides and the membrane that could prove to be particularly useful for both peptides. The second step features changes in the balance of interaction between peptides and the membrane. In this stage, the interactions between the peptides are much more effective, promoting the formation of secondary structures and intercalation in the membrane, as well as the aggregation of the peptide. Moreover, the last stage describes the creation of pores in the membrane. Here we demonstrate that the number of phenylalanine and arginine residues plays an essential role in membrane interaction by not only inducing the area expansion of the membrane but also forming $\beta$-sheet structures in its interface, as well as self-assembling into tubular pore structures.

\section{MATERIALS AND METHODS}

\subsection{Materials}

The lipid membrane was composed of dipalmitoylphosphatidylcholine (DPPC) (Sigma Aldrich), with a molar mass of the purchased product $734 \mathrm{~g} / \mathrm{mol}$ was used without further purification. The peptides $[\mathrm{RF}]$ and $[\mathrm{RF}]_{4}$ were synthesized in our laboratory using standard solid-phase methods and the characterization by liquid chromatography-mass spectrometry (LC-MS) provided $\mathrm{M}_{\mathrm{w}}=322.2 \mathrm{~g} / \mathrm{mol}$ (calculated: $321.4 \mathrm{~g} / \mathrm{mol}$ ) with purity = $92 \%$ for $[\mathrm{RF}]$ and $\mathrm{M}_{\mathrm{w}}=1232.6 \mathrm{~g} / \mathrm{mol}$ (calculated: $1231.6 \mathrm{~g} / \mathrm{mol}$ ) with purity $=98.5 \%$ for $[\mathrm{RF}]_{4}$. For Langmuir trough experiments, the DPPC and peptides were dissolved in chloroform and methanol, respectively, to give $1 \mathrm{mg} / \mathrm{mL}$ stock solutions. To prepare the samples for X-ray experiments, the DPPC and peptide were dissolved in 1:1 (v/v) chloroform/methanol mixtures, and the organic solvents were evaporated under vacuum for around 5 hours. Vesicle solutions were prepared by re-suspending dry films at room temperature in ultrapure water (> $18 \mathrm{M} \Omega$ ) at $10 \mathrm{mM}$. In this study, the peptide/lipid molar ratio $(\mathrm{P} / \mathrm{L})$ ranged from 0 up to 0.1 .

\subsection{Langmuir-Blodgett (LB)}

For the study of the interaction between DPPC and $[R F]_{n}$, ultrapure water was used to prepare the subphase in the LB trough from KSV NIMA (Biolin Scientific, Finland) at 21 ${ }^{\circ} \mathrm{C}$. The lipid and peptide solutions with different $\mathrm{P} / \mathrm{L}$ ratios were mixed at room temperature to achieve homogenization for 30 minutes. Afterward, the mixture was spread at the water-air interface of the trough and left to equilibrate for a minimum of 15 
min to allow for organic solvent evaporation. The $[\mathrm{RF}]_{\mathrm{n}} / \mathrm{DPPC}$ films were compressed at a speed of $15 \mathrm{~cm}^{2} / \mathrm{min}$ to collect pressure-area isotherms. At least six compressions were performed for each of the isotherms to observe whether their behavior was reproducible. To obtain the elastic response of the Langmuir films, the compressibility modulus $\left(C_{S}^{-1}\right)$ was calculated as follows:

$$
C_{S}^{-1}=-A \frac{\partial \Pi}{\partial A}, \quad \text { Equation } 1
$$

where A represents the average molecular area and $\Pi$ is the surface pressure data obtained from regular compression experiments.

\subsection{Atomic Force Microscopy}

The Langmuir-Blodgett (LB) transfer was implemented by lifting a small piece of mica sheet, which was washed in a sulfuric and hydrogen peroxide acid solution (3:1, v/v), at a speed of $1 \mathrm{~mm} / \mathrm{min}$. During the LB transfer, the surface pressure of the monolayer was rigorously maintained at a constant value. The mica substrate was pulled out of the solution to obtain a lipid film with hydrophobic tails oriented toward the air phase. For unsupported samples, droplets were deposited on the mica substrate and left to dry overnight in a desiccator. The AFM setup was described previously. ${ }^{20}$

\subsection{Small-Angle X-Ray Scattering (SAXS)}

SAXS data were obtained using a Bruker Nanostar instrument (with optimized optics by Xenocs) at the Institute of Physics of the University of São Paulo. The samples were placed in reusable quartz capillaries, $1.5 \mathrm{~mm}$ in diameter, mounted on stainless steel cases, permitting the measurements of the sample and buffer under same conditions. The experimentally accessible range of the modulus of the transfer moment vector $q=$ $(4 \pi \sin \theta) / \lambda$, where $\lambda$ is the radiation wavelength and $2 \theta$ is the scattering angle, was 0.18 $<q<3.5 \mathrm{~nm}^{-1}$. The description of data treatment, as well as the model used to fit the experimental data, were as described previously. ${ }^{16}$

\section{RESULTS AND DISCUSSION}

\subsection{Langmuir Isotherm Assays: Probing the Elastic Properties of Peptide/Membrane Systems}

To investigate the incorporation of peptides in membranes, we have studied four peptide/lipid ratios, within a range from 0.01 to 0.1 . The isotherms for DPPC and peptides 
at different P/L ratios are shown in Figures 1a and 1c. For DPPC monolayers, the isotherm (black line) presents a typical behavior for this lipid. ${ }^{21,22}$ The transition from liquidexpanded (LE) to liquid-condensed (LC) phases is found around $5 \mathrm{mN} / \mathrm{m}$, being followed by the monolayer collapse close to $58 \mathrm{mN} / \mathrm{m}^{22}$

The profile of isotherms upon incorporation of $[\mathrm{RF}]$ remains the same as that obtained for the DPPC system. However, upon inclusion of peptides, the molecular area is observed to grow proportionally to the increase of $\mathrm{P} / \mathrm{L}$ ratio, reaching values $\sim 100 \AA^{2}$ (Figure 1a) and the membrane collapse pressure remains unchanged.

For the system containing $[\mathrm{RF}]_{4}$, changes are observed in the profiles of isotherms (Figure 1c). Firstly, we can see an increase of $20 \AA^{2}$ in the molecular area upon addition of a small amount of peptide $(\mathrm{P} / \mathrm{L}=0.01)$. At the highest $\mathrm{P} / \mathrm{L}$ ratio studied here $(\mathrm{P} / \mathrm{L}=0.1)$, the attained values of molecular areas are up to $\sim 180 \AA^{2}$. In addition to these changes in isotherms, collapse pressures of lipid monolayers decreased, reaching values between 43 and $55 \mathrm{mN} / \mathrm{m}$.

For the precise determination of the LE-LC phase transition, we plot the dependency of the compression modulus (Equation 1) on the surface pressure (Figures S1a and S1b). The LE-LC phase transition is characterized by the minimum of the function and for the pure DPPC system is clearly observed around $5 \mathrm{mN} / \mathrm{m}$. For the system containing the RF peptide for the whole series of $\mathrm{P} / \mathrm{L}$ values, this minimum is observed. However, with the increase in $\mathrm{P} / \mathrm{L}$, a displacement to higher pressures can be observed (Figure S1a). Already in the presence of $[R F]_{4}$ the transition from LE to LC phase almost vanishes at $\mathrm{P} / \mathrm{L}=0.02$ and becomes visible only for the ratio $\mathrm{P} / \mathrm{L}=0.1$, at a surface pressure about $17 \mathrm{mN} / \mathrm{m}$ (Figure S1b).

The growth of lipid molecular area can be ascribed to the incorporation of the peptides into the lipid film. ${ }^{23}$ Regarding the displacement of the LE to LC phase transition, the presence of peptides at the monolayer interface may change the local organization of lipid molecules, leading to an increase in regions that are less ordered, even at higher surface pressures, promoting more flexible membranes. Collapse pressures for monolayers in the presence of $[R F]_{4}$ are $\sim 15 \mathrm{mN} / \mathrm{m}$ lower than those obtained for the system with $[\mathrm{RF}]$. These results indicate that $[R F]_{4}$ induces more significant changes in the membrane in comparison to $[R F]$.

A systematic investigation of the influence of peptides on DPPC monolayers was carried out. Three distinct surface pressures have been chosen: $\Pi_{1}=5 \mathrm{mN} / \mathrm{m}$, related to the liquid- 
expanded to liquid-condensed phase transition in Langmuir monolayers in pure DPPC system; $\Pi_{2}=30 \mathrm{mN} / \mathrm{m}$ already known as the natural pressure of the bilayers in bulk, ${ }^{24}$ and $\Pi_{3}=40 \mathrm{mN} / \mathrm{m}$, near to the pressure range of membrane collapse. For each of the selected pressures (horizontal grey lines in Figures 1a and 1c), the corresponding molecular area is read from the isotherm curve, and the results are shown in Figures $1 \mathrm{~b}$ and $1 \mathrm{~d}$.

The molecular area of lipid monolayer at $\Pi_{1}$ is the most affected by the presence of [RF] peptide (Figure 1b). For DPPC monolayer, the molecular region starts with $44 \AA^{2}(\mathrm{P} / \mathrm{L}=$ 0.01 ) and reaches values close to $100 \AA^{2}$ at peptide/lipid ratio $\mathrm{P} / \mathrm{L}=0.1$. When lateral pressure increases to $\Pi_{2}$ in peptide-free membranes, molecular areas are about $37 \AA^{2}$ and $40 \AA$, respectively, at $\mathrm{P} / \mathrm{L}$ ratios of 0.01 and 0.1 . At pressure $\Pi_{3}$, molecular areas are slightly smaller: they start at $34 \AA^{2}$ and increase up to $37 \AA^{2}$, remaining almost unchanged for compositions above $\mathrm{P} / \mathrm{L}=0.05$.

The molecular area as a function of $\mathrm{P} / \mathrm{L}$ for $[\mathrm{RF}]_{4}$ peptides is plotted in Figure 1d. At surface pressure $\Pi_{1}$, we observe a significant increase in the molecular area upon addition of peptide. Molecular areas increase from $43 \AA^{2}$ (DPPC) to $58 \AA^{2}$ at $\mathrm{P} / \mathrm{L}=0.01$, and reach values close to $120 \AA^{2}$ at $\mathrm{P} / \mathrm{L}=0.1$. An interesting behavior is observed for surface pressures $\Pi_{2}$ and $\Pi_{3}$ : molecular areas present a minimum at $\mathrm{P} / \mathrm{L}=0.02$ which is followed by a growth at higher $\mathrm{P} / \mathrm{L}$ ratios, indicating two regimes and multistate interactions between peptides and membranes. ${ }^{19}$

As a whole, the presence of amyloid-like peptides leads to growth of molecular area at all pressures investigated here. Since molecular areas are bigger by $\sim 10 \AA^{2}$ in samples containing $[R F]_{4}$, it is likely that larger aggregates are present at the DPPC interface when this peptide is embedded in the membranes. 

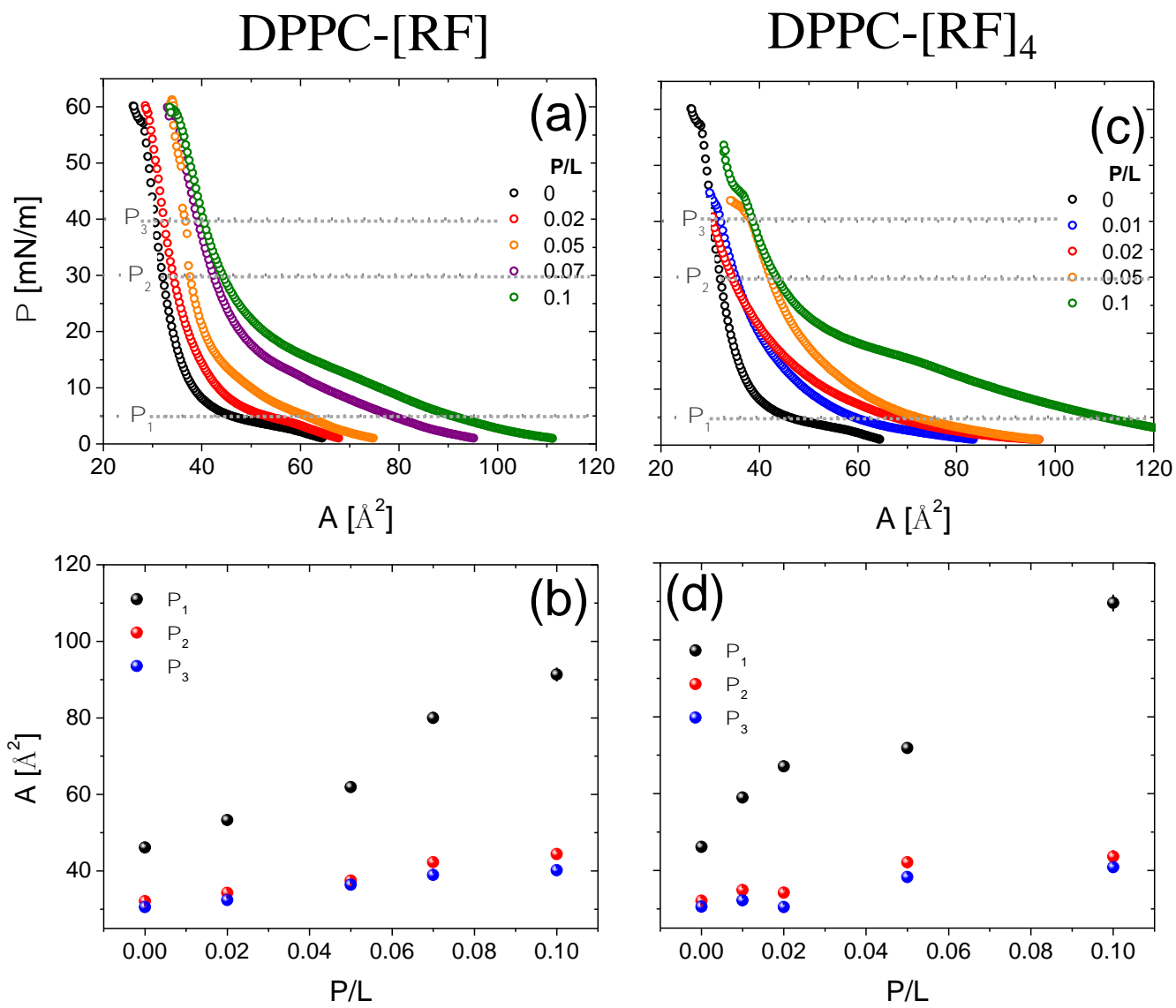

Figure 1. Surface pressure isotherms for DPPC with different ratios of the peptide (a) [RF] and (c) $[\mathrm{RF}]_{4}$; The molecular area at the three surface pressures as a function of P/L (b) $[\mathrm{RF}]$ and $(\mathrm{d})[\mathrm{RF}]_{4}$.

It is also possible to distinguish organization of lipid molecules and elastic behavior of monolayers by investigating the compressibility modulus $\left(C_{S^{-1}}^{-1}\right)$. For DPPC monolayers, the liquid-expanded (LE) phase is characterized by $C_{S}^{-1}$ values between 12.5 and $50 \mathrm{mN} / \mathrm{m}$, while for the liquid-condensed phase (LC) this parameter is in the range $100<C_{S}^{-1}<250$ $\mathrm{mN} / \mathrm{m}$ (interval indicated by dashed lines in Figure $\mathrm{S} 2$ ). ${ }^{25}$

The compressibility modulus $\left(C_{S}^{-1}\right)$ was calculated according to equation 1 , and the behavior of $C_{S}^{-1}$ as a function of molecular area for both peptides is shown in Figure S2. Increasing the amount of $[R F]$ in the lipid monolayer leads to a continuous decrease of the compressibility modulus (Figure S2a), with the minimum value being observed at $C_{S}^{-1}=$ $150 \mathrm{mN} / \mathrm{m}$, characteristic of liquid-condensed phase. The decrease of $C_{S}^{-1}$ is an indication that the lipid system is softened upon incorporation of peptide. ${ }^{25}$

In the case of DPPC monolayers in the presence of $[\mathrm{RF}]_{4}$, lower values for $C_{S}^{-1}$ are observed (Figure $\mathrm{S} 2 \mathrm{~b}$ ). For the composition $\mathrm{P} / \mathrm{L}=0.01$, a peak value near to $130 \mathrm{mN} / \mathrm{m}$ is 
observed, characteristic of a much less rigid LC phase. The lowest value of $C_{S}^{-1}$ was obtained at $\mathrm{P} / \mathrm{L}=0.02(\sim 85 \mathrm{mN} / \mathrm{m})$, and the monolayer in this condition is not characterized as a condensed liquid phase. ${ }^{25}$ On the other hand, for $\mathrm{P} / \mathrm{L}=0.05$, the compressibility modulus increases again, and a shift in the peak from 30 to $45 \AA^{2}$ is observed. The growth of molecular area indicates that the peptide is incorporated in the membrane, triggering a drastic change in lipid-molecule organization. At the largest molar ratio $(P / L=0.1)$, a different behavior for compressibility appears, and two peaks at $32 \AA^{2}$ and $40 \AA^{2}$ are observed with $C_{S}^{-1}$ values close to $120 \mathrm{mN} / \mathrm{m}$, typical of a LC phase. These two peaks suggest the coexistence between two phases, one of pure lipid (32 $\left.\AA^{2}\right)$ and another containing peptide $\left(40 \AA^{2}\right)$.

In both cases, the presence of the peptides changes the compressibility of membranes. However, the system with $[R F]_{4}$ shows a stronger interaction which is likely responsible for the LC-to-LE transition in membranes.

\subsection{Atomic Force Microscopy Assays: ultrastructural information from membrane interfaces}

The presence of arginine in the peptide sequences may increase interfacial interactions with the zwitterionic membrane surface due to hydrogen-bonding interactions, ${ }^{1}$ and this effect can enhance the carpet effect of membrane disruption. Since $[R F]_{4}$ also contains multiple phenylalanine residues, these could be interleaved in the membrane, destabilizing the membrane locally. ${ }^{2,26}$ These mechanisms may be associated with an increase in molecular areas and changes in elasticity in the monolayers.

Such interfacial effects on lipid monolayers can be monitored using the AFM technique associated with the Langmuir-Blodgett (LB) methodology, as shown in Figure 2. Such microscopy experiments were carried out at the three surface pressure regions of interest $\left(\Pi_{1}, \Pi_{2}\right.$ and $\left.\Pi_{3}\right)$ for either pure DPPC layers or peptide-lipid mixtures $(\mathrm{P} / \mathrm{L}=0.1)$.

An average height around $1.5 \mathrm{~nm}$ (Figure 2a) has been obtained in pure DPPC films, which is compatible with previous results in the literature. ${ }^{27,}{ }^{28}$ When the monolayer is exposed to the lowest pressure $\left(\Pi_{1}\right)$ (Figure 2a), small lipid clusters are observed, corresponding to LE phase domains. ${ }^{28}$ At surface pressure $\Pi_{2}$, large lipid clusters from regions of the LC phase and small LE domains are seen (Figure 2b). In the third region, near to the collapse of the DPPC membrane (Figure 2c), a well-organized film of completely closed hexagon-like figures is observed. 
Upon incorporation of $[\mathrm{RF}](\mathrm{P} / \mathrm{L}=0.1)$, agglomerates increased in both number and size, consistent with results from isotherm measurements that showed a growth of the molecular area in the LE to LC phase-transition region (Figure 2d). At surface pressure $\Pi_{2}$, the AFM image shown in Figure 2e reveals a modification in the organization of the lipid film and coexistence between LC ( 3 $\mu$ m-diameter cluster) and LE ( 300 nm-diameter cluster) phases. At surface pressure $\Pi_{3}$, pores of the order of $10 \mathrm{~nm}$ in diameter are observed, as shown in Figure 2f.

The images for the system containing $[R F]_{4}$ are exhibited in Figures $2 \mathrm{~g}$ and $2 \mathrm{~h}$. At $\Pi_{1}$, the formation of large $\sim 2 \mu \mathrm{m}$ DPPC-peptide clusters were observed. At surface pressure $\Pi_{2}$, much larger pores are identified, most of which reaching about $400 \mathrm{~nm}$ diameter.

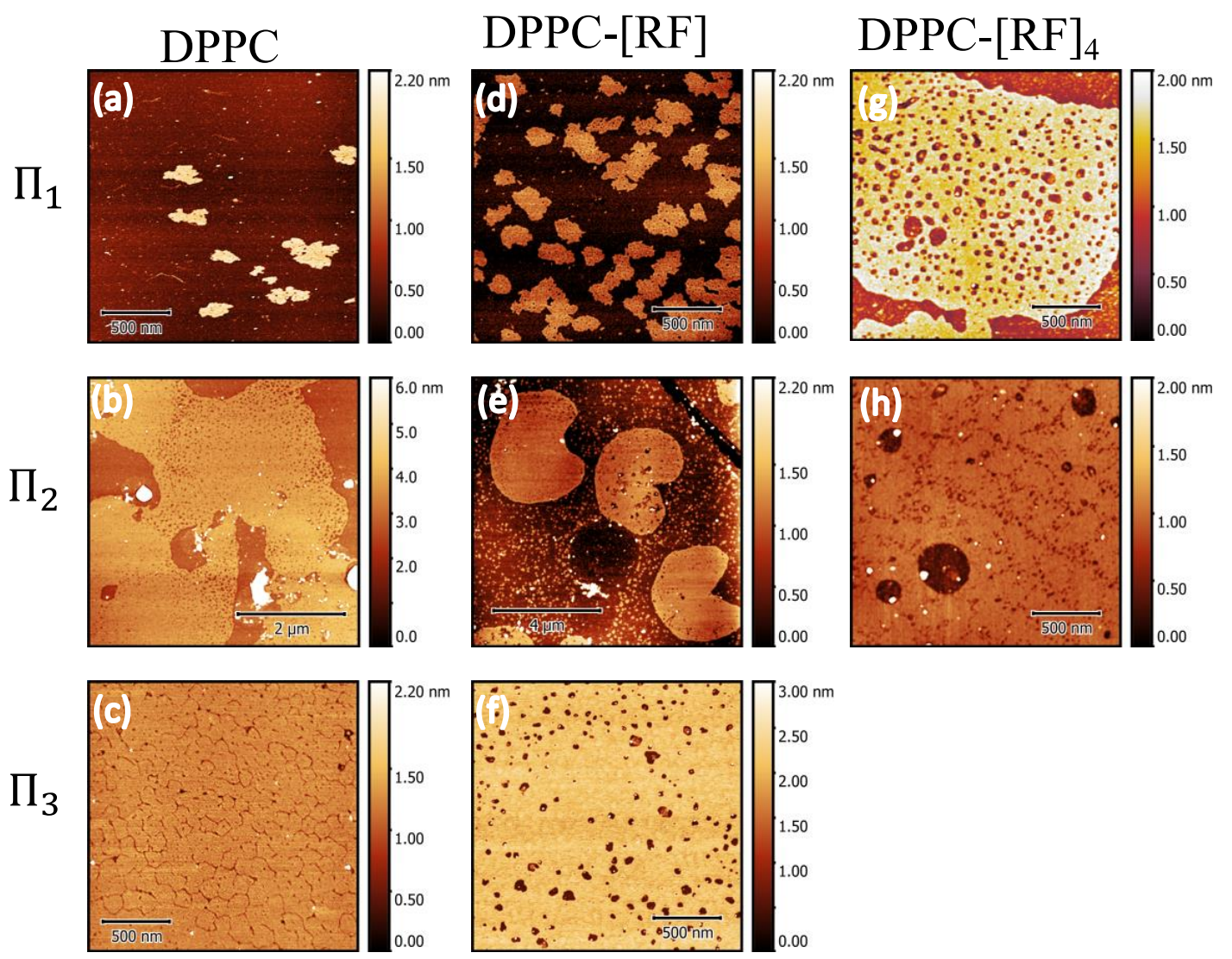

Figure 2. AFM images obtained by exposure of the hydrophobic regions in LB films under different lateral pressures for (a-c) pure DPPC; (d-f) DPPC-[RF]; (g-h) DPPC-[RF] ${ }_{4}$

The incorporation of peptides to the membrane has triggered some changes in the lipid film structures. A noteworthy point is the increase in the size of the agglomerates at low pressures, which correlates well with molecular area data obtained from isotherm analyses. The second interesting finding is the increase of the LE domains at a pressure of $30 \mathrm{mN} / \mathrm{m}$, which indicates both the presence of less ordered regions in the membrane and low lateral 
compressibility of the system. Finally, we observe a higher number of pores in the membrane, as well as increase of pore size, in the presence of $[R F]$ or $[R F]_{4}$ when compared with pure DPPC films.

The same experiments were conducted using a hydrophilic coverslip as substrate for LB films. In this case, a second transference was performed on top of the previous monolayer, to observe the peptide aggregates on bilayer surfaces. Figure S3a shows that the number of clusters increases when $[R F]$ was incorporated in the bilayer at $\Pi_{1}$. Also, small aggregates of fibrillar and spherical structures are observed, with typical heights at 3 and $6 \mathrm{~nm}$, respectively. Figure S3b exhibits a better-organized system of $\sim 5 \mathrm{~nm}$ in height, large spherical aggregates of $\sim 40 \mathrm{~nm}$, and long fibers of $\sim 12 \mathrm{~nm}$ at surface pressure $\Pi_{2}$. In the $\mathrm{DPPC} /[\mathrm{RF}]_{4}$ system, a high number of fiber fragments $\sim 8 \mathrm{~nm}$ in height are observed on the membrane at surface pressure $\Pi_{1}$, as seen in Figure $\mathrm{S} 3 \mathrm{c}$. At $\Pi_{2}$, both fragments of the lipid layers mixed with $[\mathrm{RF}]_{4}$ are observed (Figure S3d).

\subsection{Small-Angle X-ray Scattering: Nanoscopic Structure under Hydrated Conditions}

Experiments with DPPC vesicles were performed in aqueous solutions to investigate the global morphological and structural changes in the membrane with different peptide concentrations, as an environment of greater complexity could lead to pore formation in lipid layers. Structural aspects of the vesicles and the influence of peptides in these bilayers can be monitored using the SAXS technique. Figures $3 \mathrm{a}$ and $3 \mathrm{~b}$ show the scattered intensity, I(q), as a function of the scattering vector for $[R F]$ and $[R F]_{4}$, respectively.

In samples containing only DPPC bilayers $(\mathrm{P} / \mathrm{L}=0)$, the data are featured by two peaks at $\mathrm{q}=1 \mathrm{~nm}^{-1}$ and $\mathrm{q}=2 \mathrm{~nm}^{-1}$, indicating the presence of multilamellar vesicles with a repeat distance of $\sim 6.3 \mathrm{~nm}$, in agreement with results from literature. ${ }^{29}$ The addition of peptides to the mixture strongly affects the degree of order in the vesicles, as attested by progressive disappearance of peaks in the scattering curves. In samples containing $\mathrm{P} / \mathrm{L} \geq 0.02$, the scattering profiles are characterized by one diffuse bump at intermediate range, which is characteristic of unilamellar vesicles. 

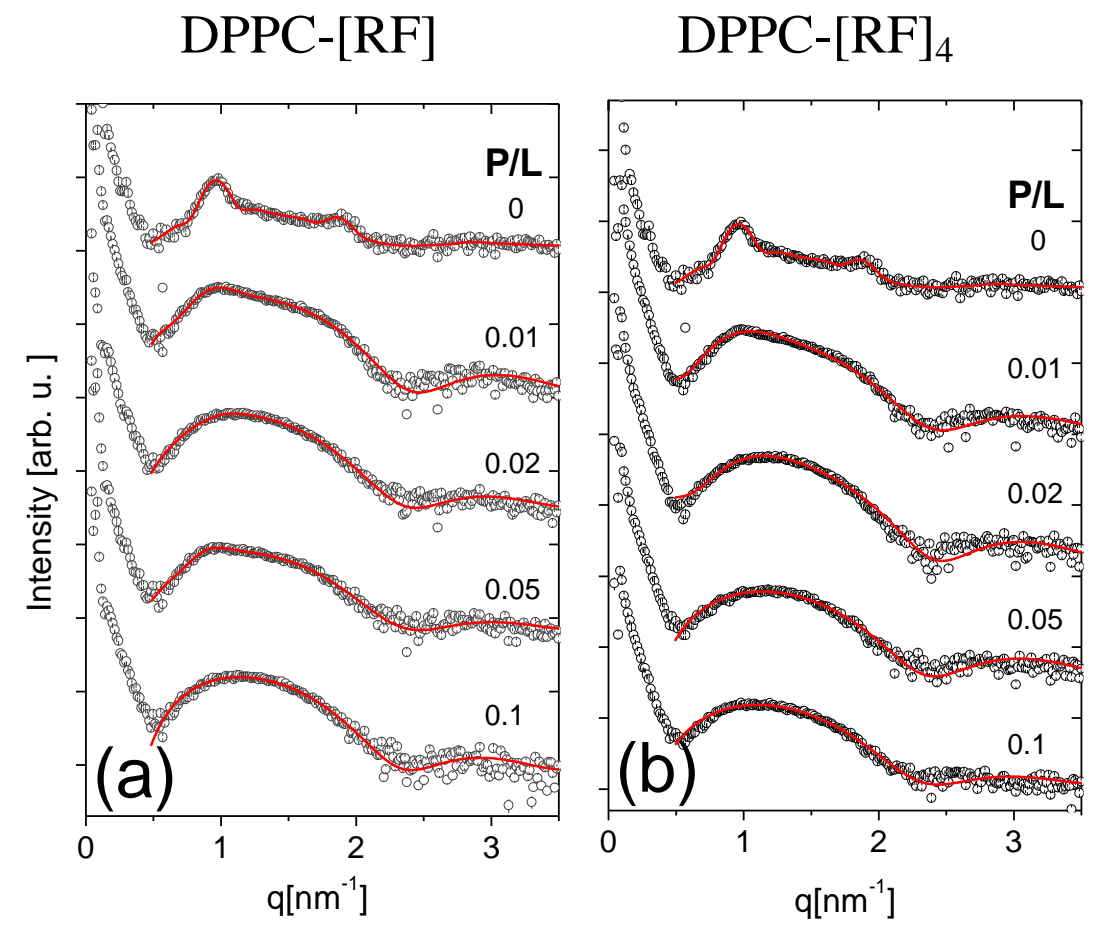

Figure 3. X-ray scattering for different $P / L$ ratios from 0 to 0.1 for (a) $[R F]$ and (b) $[R F]_{4}$ sequences. The solid lines represent the fitting to the experimental data using the Gaussian model.

The Inverse Fourier transform (IFT) method, which enables the determination of pair distance distribution, $p(r)$, has been used to show that the systems with the absence of peaks are consistent with unilamellar vesicles. ${ }^{30}$ Figure 4 a displays the function $p(r)$ in the region $0<r<4 \mathrm{~nm}$, the change of the signature of the electron density contrast is of a bilayer, followed by a negative valley related to the central hydrophobic part of the membrane. It is also possible to determine the maximum diameter of the vesicles by using the $p(r)$ function. For both the $[R F]$ and $[R F]_{4}$ systems, the larger the P/L, the greater the size of the vesicles in the solution (Figure 4a). However, the maximum unilamellar vesicle radius achieved in the $[R F]$ system at a $P / L=0.1$ molar ratio is smaller than in the $[R F]_{4}$ system at $P / L=0.02$.

To extract quantitative information from SAXS data, and unveil finer features of membranes in the presence of peptides, we investigated the electron density profile of bilayers. The model proposed by Oliveira et $a l .{ }^{31}$ was used to perform full range data fitting, and the global results obtained from this approach have allowed to discriminate structural parameters such as lamellar periodicity and electronic density profile, as well as the Caillé parameter, which is related to the membrane flexibility. 
The electron contrast density profiles for both peptides are presented in Figure 4b. The negative region of $\Delta \rho$ is altered upon incorporation of peptides, suggesting a possible change in the organization of the lipid aliphatic chains.

The thickness of the bilayer membrane can be determined using the electron contrast profiles. ${ }^{16,31}$ The thickness value of $4.8 \mathrm{~nm}$ has been found in the pure DPPC system, which is compatible with values reported in the literature. ${ }^{32}$

An increase in the thickness of the bilayer is observed when peptides are incorporated into the membranes, indicating the presence of the peptides at the membrane interface (Figure 4c).

The Caillé parameter $(\eta)$ obtained for multilamellar vesicles is shown in Table 1 . The value of $\eta$ obtained for pure DPPC was $\sim 0.07$, while the value for lipid-peptide systems ranges from 0.5 to 1 , indicating greater flexibility of the membrane. These results corroborate the behavior of the lateral compressibility described in Figure S2 for the LB film, which suggests a more flexible system with the incorporation of the peptide to the membrane.
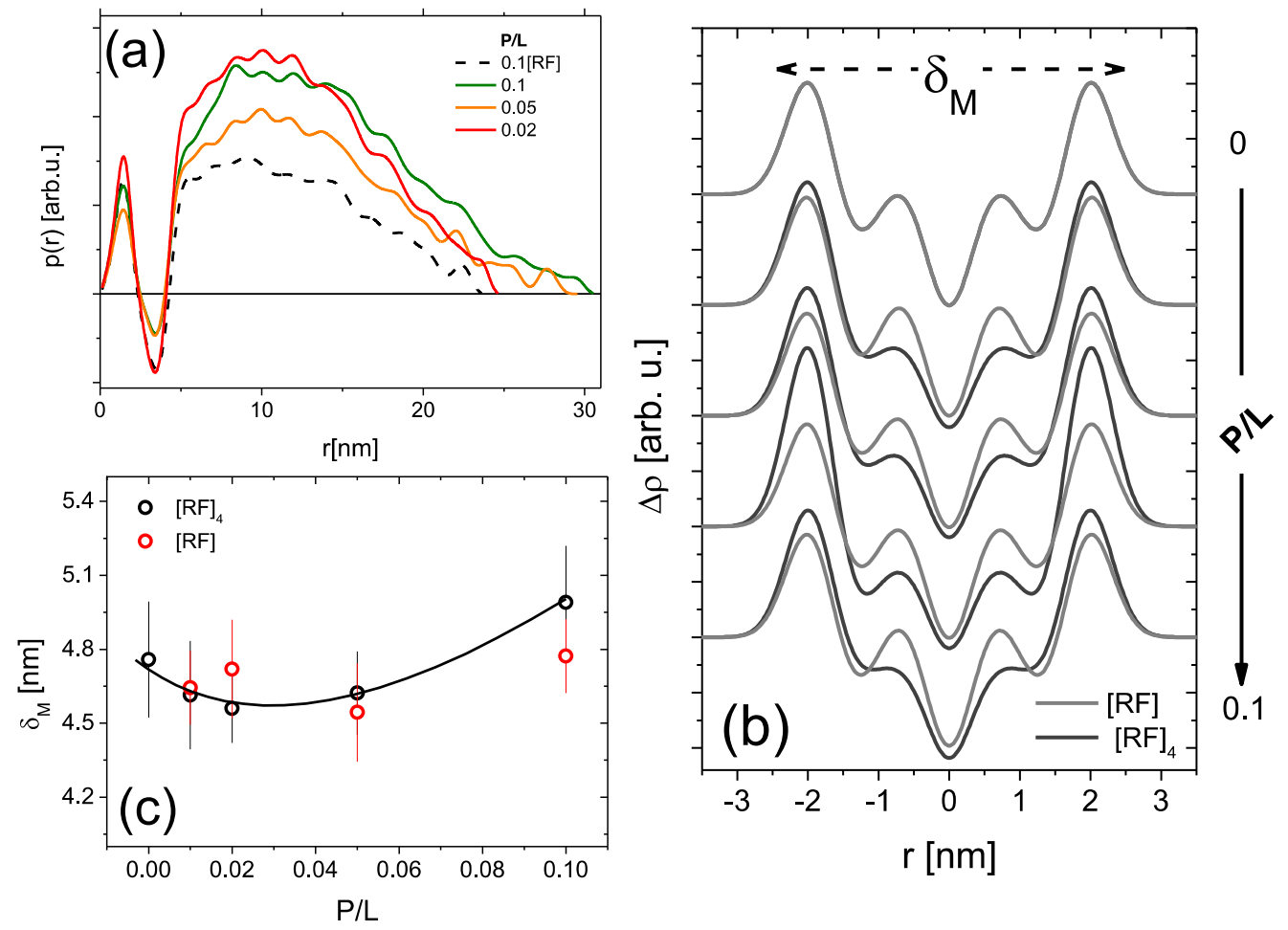

Figure 4. (a) Plot of the $p(r)$ function, where the continuous lines correspond to the $[\mathrm{RF}]_{4}-$ DPPC system data and the dotted one to the $[R F]-D P P C$ system for $\mathrm{P} / \mathrm{L}=0.1$. Structural parameters obtained from the scattering data in Figure 3, (b) membrane thickness $\left(\delta_{M}\right)-$ the lines are guides to the eyes, and (c) the electron density profile $(\Delta \rho)$. 
Table 1. Caillé parameter obtained for DPPC multilamellar vesicles with a variable $\mathrm{P} / \mathrm{L}$ ratio (Figure 2) using the Gaussian Deconvolution Method. For unilamellar vesicles (N close to 1$), \eta$ is not applicable ("N/A").

\begin{tabular}{lll}
\hline \hline $\mathbf{P} / \mathbf{L}$ & {$[\mathbf{R F}]$} & {$[\mathbf{R F}] \mathbf{4}$} \\
\hline 0 & $0.07 \pm 0.02$ & $0.07 \pm 0.02$ \\
0.01 & $0.53 \pm 0.12$ & $1.08 \pm 0.15$ \\
0.02 & $1.32 \pm 0.42$ & N/A \\
0.05 & $0.88 \pm 0.21$ & N/A \\
0.1 & N/A & N/A \\
\hline \hline
\end{tabular}

Multi-to-unilamellar transitions of lipid vesicles in the presence of short peptides have already been observed previously. ${ }^{16,33}$ For lipid systems with uncharged peptide systems containing phenylalanine $(\mathrm{F})$ and cysteine $(\mathrm{C})$, the transition occurs at ratios of $\mathrm{P} / \mathrm{L}>0.1$ for $\mathrm{FF}$ and in $\mathrm{P} / \mathrm{L}>0.01$ for $\mathrm{CFF}$, indicating that cysteine residues interact much more strongly with the membrane and is essential for the transition. ${ }^{16}$ For $\mathrm{R}_{3} \mathrm{~F}_{3}$ and $\mathrm{R}_{4} \mathrm{~F}_{4}$ in the presence of DPPG/DPPE mixed membranes, SAXS data showed that the transition occurs at $\mathrm{P} / \mathrm{L}<0.16 .{ }^{33}$ In the present work, we observed that the transitions for $[\mathrm{RF}]$ occur at the same ratio as observed for $\mathrm{FF}$, indicating low interaction with the membrane. On the other hand, there is more significant interaction with the DPPC layers for the $[R F]_{4}$ system, as a more significant number of arginine residues is present in the peptide, promoting substantial structural changes.

\subsection{Atomic Force Microscopy Assays: Unsupported vesicle system}

Figure 5 presents AFM images of pure DPPC spherical structures of $\sim 80 \mathrm{~nm}$ in size with low polydispersity. In some cases, an overlap of layers can be noted, which is typical of multilamellar systems (Figure 5a). For lipid- $[R F]$ complexes $(P / L=0.01)$, it was possible to observe multilamellar vesicles, as shown in Figure $5 \mathrm{~b}$. Such vesicles were much bigger than the ones present in the pure DPPC system, corroborating the behavior of the maximum diameter of the vesicles obtained by the $p(r)$ function, as shown in Figure 4a. Remarkably, the images for the composition $\mathrm{P} / \mathrm{L}=0.1$ (Figure $5 \mathrm{c}$ ) show that this system presents unilamellar vesicles that are $\sim 40$ times larger than in the $\mathrm{P} / \mathrm{L}=0.01$ ratio. The white feature in the upper right corner of the figure is the peptides aggregated on the DPPC vesicles (see insert Figure S5), where we observe fibrillar structures with $\sim 30 \mathrm{~nm}$ high. AFM also reveals 
the presence of pores of $\sim 50 \mathrm{~nm}$ in size that have the same behavior observed in the AFM images for LB films (Figure 2f).

Figure $5 \mathrm{~d}$ shows images of vesicles for $\mathrm{DPPC}-[\mathrm{RF}]_{4}$ at $\mathrm{P} / \mathrm{L}=0.01$, featuring multilamellar vesicles larger than those observed for both pure DPPC and the lipid-[RF] system. Also, for both lipid-peptide samples at a larger P/L ratio, only a large membrane agglomerate was observed, indicating a vesicle fusion pathway. It is interesting to observe the large plate that features pores with up to $1 \mu \mathrm{m}$ in diameter at $\mathrm{P} / \mathrm{L}=0.1$ in this system in Figure 5e. The presence of such pores may relate to the significant increase in the Caillé parameter found in the SAXS data. These results are compatible with those previously presented for lipid monolayers, indicating that the behavior observed in the lipid monolayers also occurs in the unsupported system.

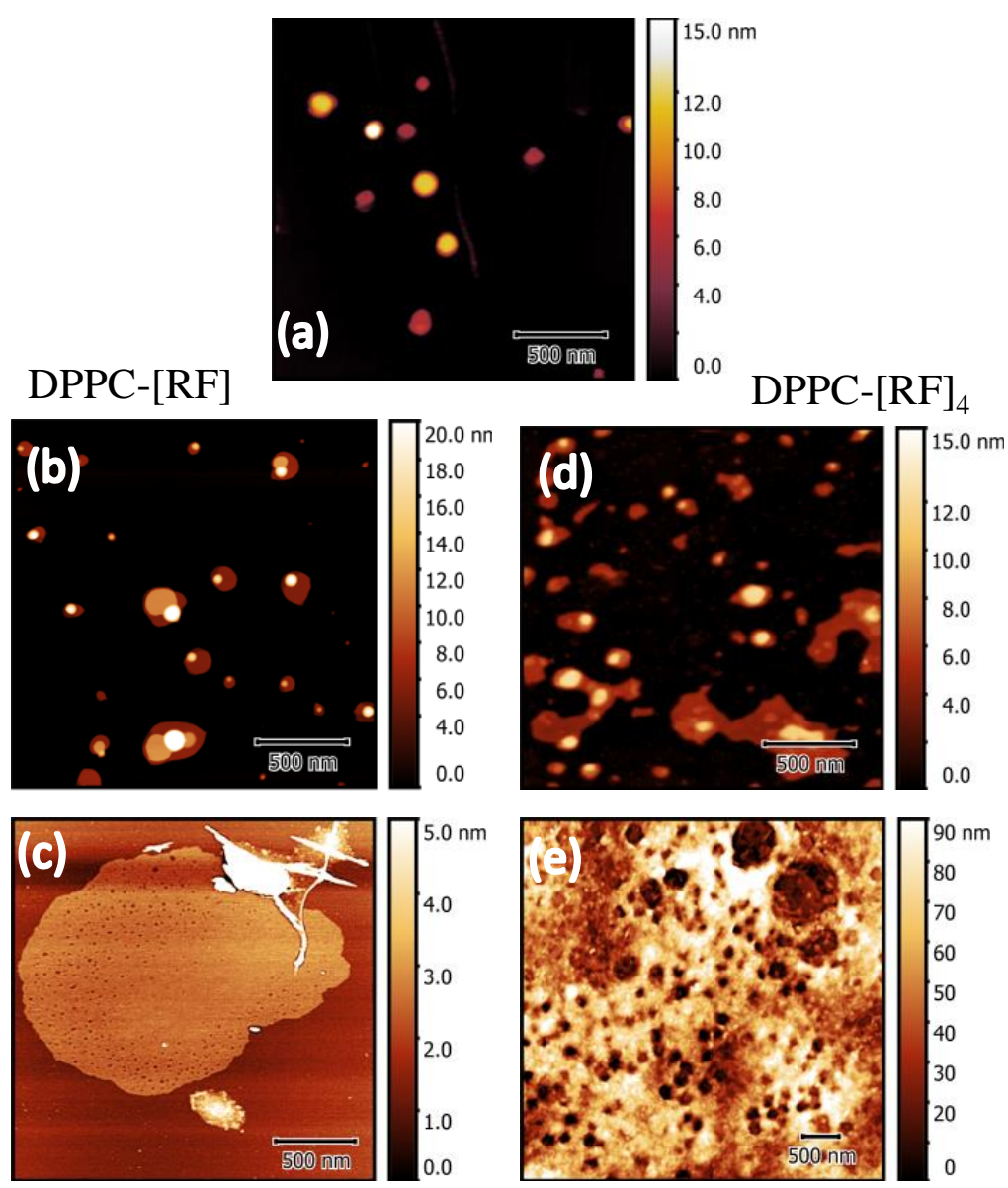

Figure 5. AFM images for unsupported samples in aqueous solution: a) pure DPPC; DPPC$[R F]$ for different ratios of P/L (b) 0.01 and (c) 0.1 ; DPPC-[RF $]_{4}$ for P/L (d) 0.01 and (e) 0.1 . 


\subsection{Interaction Model for Peptide/Membrane Systems}

All the experimental evidence presented in this work unequivocally demonstrates strong interaction between the short peptides based on the $[R F]$ pair and lipid membranes. The adsorption isotherm measurements indicate a multi-stage lipid-peptide interaction process, ${ }^{5,19,34}$ which can be described by the three-interaction-stage mechanism proposed by Hirst and collaborators that involves surface building, insertion, and expansion. ${ }^{19}$ The surface building stage corresponds to the first accumulation of the peptide at the lipid interface. Literature studies propose that the presence of peptides in lipid membranes promotes significant local changes in the organization of lipid aliphatic chains. ${ }^{5,35}$ The DPPC layer is in its gel phase $\left(L_{\beta}\right)$ at a temperature of $21^{\circ} \mathrm{C}$, which implies a rigid membrane containing stretched aliphatic chains (Figure 6a). However, the presence of the peptide induces a transition from a gel to a fluid-phase for the DPPC layers $\left(L_{\alpha}\right)$, (Figure 6b) therefore decreasing the local stiffness of the membrane. ${ }^{4,36}$

This process can explain not only the significant increase in the molecular area that occurred in the presence of both peptides but also the decrease of the membrane compressibility factor. It also leads to the change of electron density contrast in the region of the aliphatic tails, which is interpreted as a phase transition from $L_{\alpha}$ to $L_{\beta}$. The second step was the insertion of the peptides into the membrane. A continuous increase in the [RF] molecular area was observed with the intercalation of the peptide into the DPPC layers. On the other hand, when increasing the concentration of the $[R F]_{4}$ peptides, the molecular area stabilizes at intermediate values of $\mathrm{P} / \mathrm{L}$ (around $\mathrm{P} / \mathrm{L}=0.02$ ). This may indicate that the peptide is intercalated into the membrane, ${ }^{5,35-37}$ decreasing the peptide-lipid interaction, locally increasing the peptide-peptide affinity, and promoting secondary structure formation (Figure 6c).

CD spectra presented in Figure S4 show two positive bands, one located at $\sim 195 \mathrm{~nm}$, correlated with antiparallel $\beta$-sheet conformation, ${ }^{20}$ and the other at $219 \mathrm{~nm}$, characteristic of phenylalanine $n-\pi^{*}$ electronic transitions. ${ }^{38}$ In contrast, the DPPC vesicles alone do not show any CD signal. Also, we observed a red-shift in the wavelength of absorption for $[R F]_{4}$ peptides, suggesting that $\beta$-sheet content is enhanced as the number of $[R F]$ units increases in the sequences. The results indicate that interaction with membranes may induce structuring and lead to secondary structure features that are not present in aqueous solutions. Notably, they suggest that morphological changes related to the formation of $\beta$ sheet and $\beta$-turn type structures may be associated with pore formation. 
The effective pore structuration occurs at the expansion stage. The AFM images show such formation in the membrane for both systems, which can be justified by the considerable increase of both the molecular area in the isotherm experiment and the flexibility observed in LB films and vesicle solutions (Figures 6d and 6e).

(a)

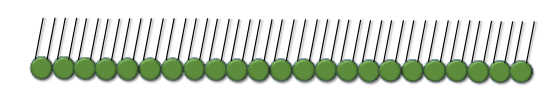

(b)

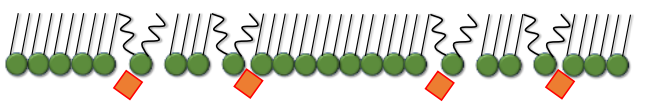

(c)

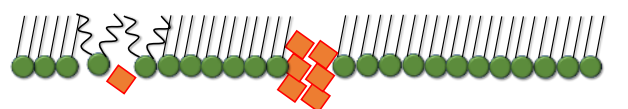

(d)

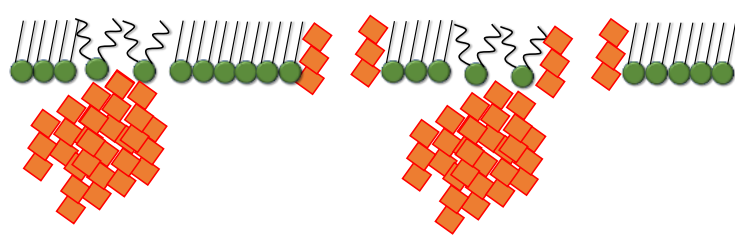

(e)

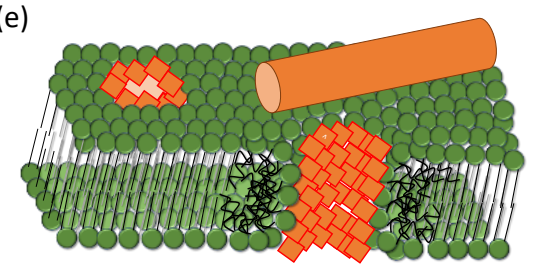

Figure 6. Schematic depicting peptide-lipid interactions: (a) monolayer of DPPC, (b) surface binding, (c) insertion, (d) expansion, and (e) 3D representation of pore formation.

\section{CONCLUSIONS}

The present study investigated the interaction of short peptides based on the pair [RF] with DPPC phospholipid membranes, both as LB films and vesicle solutions. Arginineenriched sequences have attracted increasing interest due to their extensive presence in bioactive peptide classes such as antimicrobial and cell penetrating peptides. On the other hand, structural motifs usually found in amyloid-forming sequences often display aromatic residues that conveys directionality for fibrillization through $\pi$-stacking interactions.

Therefore, the sequences investigated here are archetypical models to discriminate the role of these amino acid residues on the interaction of these major peptide classes with cell membranes. For the $[R F]$ sequence, such interactions promote a continuous increase of the molecular area, evidencing affinity of this peptide to membrane interfaces. On the other hand, for $[\mathrm{RF}]_{4}$ a more complex behavior is observed since the molecular area does not show a smooth increase with peptide concentration as it is incorporated in the DPPC layer, which promotes the reduction of compressibility and leads to a more flexible membrane.

Morphological changes in the DPPC vesicles were observed in the presence of peptides, which were monitored by SAXS. The first finding was the transition of MLV to ULV systems for different $\mathrm{P} / \mathrm{L}$ ratios. Such a transition occurs at lower $\mathrm{P} / \mathrm{L}$ for $[\mathrm{RF}]_{4}$ than for the 
[RF] system, indicating that the increased number of hydrophobic residues promotes a better interaction with the zwitterionic membrane surface. The arginine group may also interact hydrophilically with DPPC by hydrogen bonding and, consequently, locally destabilize it. The electron contrast density in the hydrophobic region is related to the phase transition from gel to fluid of aliphatic lipid chains, which also leads to a decrease of the lateral compressibility modulus and an increased Caillé parameter.

AFM images depicted not only the formation of peptide fibers or spherical clusters on the surface of the lipid but also the rupture of the membrane at higher pressures. Both a significant increase in vesicle size and the transition from MLV to ULV were observed in the unsupported system. The images also showed the presence of pores through the lipid bilayer, suggesting that the phenylalanine residues seem to have a significant effect on both pore size and membrane structure.

\section{SUPPORTING INFORMATION}

Supporting information: The determination of the LC-LE phase transition, in-plane elasticity $\left(\mathbf{C}_{\mathbf{S}}^{-\mathbf{1}}\right)$ for DPPC monolayer and different P/L ratios. AFM images of samples under different lateral pressures and CD spectra for lipid-peptide complexes.

\section{CONFLICT OF INTEREST}

The authors declare that they have no known competing financial interests or personal relationships in this work.

\section{ACKNOWLEDGEMENTS}

This work was supported by FAPESP (grant no. 2017/02317-2) and CNPq (grant no. 304389/2019-6). INCT in Bioanalytics (FAPESP grant no. 2014/50867-3 and CNPq grant no. 465389/2014-7) is kindly acknowledged for the grants. BBG acknowledge FAPESP (project number 2018/05888-3) for a post-doctoral fellowship. CLPO is supported by FAPESP (2018/16092-5, 2017/27078-0) and CNPq (303001/2019-4). ERS is supported by FAPESP under grant 2019/20907-7. The staff at LNNano are kindly recognized for their valuable contribution with the AFM analysis.

\section{REFERENCES}


(1) Silva, E. R.; Listik, E.; Han, S. W.; Alves, W. A.; Soares, B. M.; Reza, M.; Ruokolainen, J.; Hamley, I. W. Sequence length dependence in arginine/phenylalanine oligopeptides: Implications for self-assembly and cytotoxicity. Biophys. Chem. 2018, 233, $1-12$.

(2) Stckl, M. T.; Zijlstra, N.; Subramaniam, V. alpha-Synuclein Oligomers: an Amyloid Pore? Mol. Neurobiol. 2013, 47, 613-621.

(3) Ege, C.; Lee, K. Y. C. Insertion of Alzheimer's A beta 40 peptide into lipid monolayers. Biophys. J. 2004, 87, 1732-1740.

(4) Vander Zanden, C. M.; Warnkiler, L.; Bowers, I.; Watkins, E. B.; Majewski, J.; Chi, E. Y. Fibrillar and Nonfibrillar Amyloid Beta Structures Drive Two Modes of MembraneMediated Toxicity. Langmuir 2019, 35, 16024-16036.

(5) Cuco, A.; Serro, A. P.; Farinha, J. P.; Saramago, B.; da Silva, A. G. Interaction of the Alzheimer A beta(25-35) peptide segment with model membranes. Colloids Surf., B 2016, $141,10-18$.

(6) Jang, H.; Arce, F. T.; Capone, R.; Ramachandran, S.; Lal, R.; Nussinov, R. Misfolded Amyloid Ion Channels Present Mobile beta-Sheet Subunits in Contrast to Conventional Ion Channels. Biophys. J. 2009, 97, 3029-3037.

(7) Jang, H.; Arce, F. T.; Ramachandran, S.; Capone, R.; Azimova, R.; Kagan, B. L.; Nussinov, R.; Lal, R. Truncated beta-amyloid peptide channels provide an alternative mechanism for Alzheimer's Disease and Down syndrome. Proc. Natl. Acad. Sci. U.S.A. 2010, 107, 6538-6543.

(8) Sato, H.; Felix, J. B. Peptide-membrane interactions and mechanisms of membrane destruction by amphipathic alpha-helical antimicrobial peptides. Biochim. Biophys. Acta, Biomembr. 2006, 1758, 1245-1256.

(9) Thakur, G.; Micic, M.; Leblanc, R. M. Surface chemistry of Alzheimer's disease: A Langmuir monolayer approach. Colloids Surf., B 2009, 74, 436-456.

(10) Hamley, I. W. The Amyloid Beta Peptide: A Chemist's Perspective. Role in Alzheimer's and Fibrillization. Chem. Rev. 2012, 112, 5147-5192.

(11) Cecchi, C.; Stefani, M. The amyloid-cell membrane system. The interplay between the biophysical features of oligomers/fibrils and cell membrane defines amyloid toxicity. Biophys. Chem. 2013, 182, 30-43.

(12) Relini, A.; Marano, N.; Gliozzi, A. Probing the interplay between amyloidogenic proteins and membranes using lipid monolayers and bilayers. Adv. Colloid Interface Sci. 2014, 207, 81-92. 
(13) Gerbelli, B. B.; Ly, I.; Pedemay, S.; Alves, W. A.; Oliveira, E. A. The Role of Amylogenic Fiber Aggregation on the Elasticity of a Lipid Membrane. ACS Appl. Bio Mater. 2020, 3, 815-822.

(14) Schnaider, L.; Brahmachari, S.; Schmidt, N. W.; Mensa, B.; Shaham-Niv, S.; Bychenko, D.; Adler-Abramovich, L.; Shimon, L. J. W.; Kolusheva, S.; DeGrado, W. F.; Gazit, E. Self-assembling dipeptide antibacterial nanostructures with membrane disrupting activity. Nat. Commun. 2017, 8, 1365-1375.

(15) Decandio, C. C.; Silva, E. R.; Hamley, I. W.; Castelletto, V.; Liberato, M. S.; Oliveira, V. X.; Oliveira, C. L. P.; Alves, W. A. Self-Assembly of a Designed Alternating Arginine/Phenylalanine Oligopeptide. Langmuir 2015, 31, 4513-4523.

(16) Gerbelli, B. B.; Silva, E. R.; Soares, B. M.; Alves, W. A.; Oliveira, E. A. Multilamellar-to-Unilamellar Transition Induced by Diphenylalanine in Lipid Vesicles. Langmuir 2018, 34, 2171-2179.

(17) Amit, M.; Cheng, G.; Hamley, I. W.; Ashkenasy, N. Conductance of amyloid beta based peptide filaments: structure-function relations. Soft Matter 2012, 8, 8690-8696.

(18) Adler-Abramovich, L.; Reches, M.; Sedman, V.; Allen, S.; Tendler, S.; Gazit, E. Thermal and chemical stability of diphenylalanine peptide nanotubes: Implications for nanotechnological applications. Langmuir 2006, 22, 1313-1320.

(19) Hirst, D. J.; Lee, T. H.; Swann, M. J.; Aguilar, M. I. Combined Mass and Structural Kinetic Analysis of Multistate Antimicrobial Peptide-Membrane Interactions. Anal. Chem.2013, 85, 9296-9304.

(20) Pelin, J.; Gerbelli, B. B.; Soares, B. M.; Aguilar, A. M.; Alves, W. A. Amyloidogenic model peptides as catalysts for stereoselective aldol reactions. Catal. Sci. Technol. 2019, 9, 4304-4313.

(21) Toimil, P.; Prieto, G.; Minones, J.; Sarmiento, F. A comparative study of FDPPC/DPPC mixed monolayers. Influence of subphase temperature on F-DPPC and DPPC monolayers. Phys. Chem. Chem. Phys. 2010, 12, 13323-13332.

(22) Zuo, Y. Y.; Chen, R. M.; Wang, X. J.; Yang, J. L.; Policova, Z.; Neumann, A. W. Phase Transitions in Dipalmitoylphosphatidylcholine Monolayers. Langmuir 2016, 32, 8501-8506.

(23) Rodrigues, J. C.; Caseli, L. Incorporation of bacitracin in Langmuir films of phospholipids at the air-water interface. Thin Solid Films 2017, 622, 95-103.

(24) Blume, A. Comparative-study of the phase-transitions of phospholipid-bilayers and monolayers, Biochim. Biophys. Acta 1979, 557, 32-44. 
(25) Clausell, A.; Busquets, M. A.; Pujol, M.; Alsina, A.; Cajal, Y. Polymyxin B-lipid interactions in Langmuir-Blodgett monolayers of Escherichia coli lipids: A thermodynamic and atomic force microscopy study. Biopolymers 2004, 75, 480-490.

(26) Zhang, Y. J.; Shi, J. M.; Bai, C. J.; Wang, H.; Li, H. Y.; Wu, Y.; Ji, S. R. Intramembrane Oligomerization and Extra-membrane Oligomerization of Amyloid-beta Peptide Are Competing Processes as a Result of Distinct Patterns of Motif Interplay. $J$. Biol. Chem. 2012, 287, 748-756.

(27) Diociaiuti, M.; Ruspantini, I.; Giordani, C.; Bordi, F.; Chistolini, P. Distribution of GD3 in DPPC monolayers: A thermodynamic and atomic force microscopy combined study. Biophys. J. 2004, 86, 321-328.

(28) Yang, X. M.; Xiao, D.; Xiao, S. J.; Wei, Y. Domain-structures of phospholipid monolayer Langmuir-Blodgett-films determined by atomic-force microscopy. Appl. Phys. A 1994, 59, 139-143.

(29) Kornmueller, K.; Lehofer, B.; Leitinger, G.; Amenitsch, H.; Prass1, R. Peptide selfassembly into lamellar phases and the formation of lipid-peptide nanostructures. Nano Res. 2018, 11, 913-928.

(30) Glatter, O. New method for evaluation of Small-Angle Scattering data. J. Appl. Crystallogr. 1977, 10, 415-421.

(31) Oliveira, C.; Gerbelli, B.; Silva, E.; Nallet, F.; Navailles, L.; Oliveira, E.; Pedersen, J. Gaussian deconvolution: a useful method for a form-free modeling of scattering data from mono- and multilayered planar systems. J. Appl. Crystallogr 2012, 45, 1278-1286.

(32) Farkuh, L.; Hennies, P. T.; Nunes, C.; Reis, S.; Barreiros, L.; Segundo, M.; Oseliero, P. L.; Oliveira, C. L. P.; Cassago, A.; Portugal, R. V.; Muramoto, R. A.; Carretero, G. P. B.; Schreier, S.; Chaimovich, H.; Cuccovia, I. M. Characterization of phospholipid vesicles containing lauric acid: physicochemical basis for process and product development. Heliyon 2019, 5, e02648-e02658.

(33) Edwards-Gayle, C. J. C.; Barrett, G.; Roy, S.; Castelletto, V.; Seitsonen, J.; Ruokolainen, J.; Hamley, I. W. Selective Antibacterial Activity and Lipid Membrane Interactions of Arginine-Rich Amphiphilic Peptides. ACS Appl. Bio Mater. 2020, 3, 11651175.

(34) Sciacca, M. F. M.; Kotler, S. A.; Brender, J. R.; Chen, J.; Lee, D. K.; Ramamoorthy, A. Two-Step Mechanism of Membrane Disruption by A beta through Membrane Fragmentation and Pore Formation. Biophys. J. 2012, 103, 702-710. 
(35) Chi, E. Y.; Ege, C.; Winans, A.; Majewski, J.; Wu, G. H.; Kjaer, K.; Lee, K. Y. C. Lipid membrane templates the ordering and induces the fibrillogenesis of Alzheimer's disease amyloid-beta peptide. Proteins Struct. Funct. Bioinf. 2008, 72, 1-24.

(36) Jones, E. M.; Dubey, M.; Camp, P. J.; Vernon, B. C.; Biernat, J.; Mandelkow, E.; Majewski, J.; Chi, E. Y. Interaction of Tau Protein with Model Lipid Membranes Induces Tau Structural Compaction and Membrane Disruption. Biochemistry 2012, 51 , 2539-2550. (37) Hirst, D. J.; Lee, T. H.; Pattenden, L. K.; Thomas, W. G.; Aguilar, M. I. Helix 8 of the angiotensin-II type 1A receptor interacts with phosphatidylinositol phosphates and modulates membrane insertion. Sci. Rep. 2015, 5, 9972-9986.

(38) Krysmann, M. J.; Castelletto, V.; Hamley, I. W., Fibrillisation of hydrophobically modified amyloid peptide fragments in an organic solvent. Soft Matter 2007, 3, 1401-1406. 
TOC ENTRY

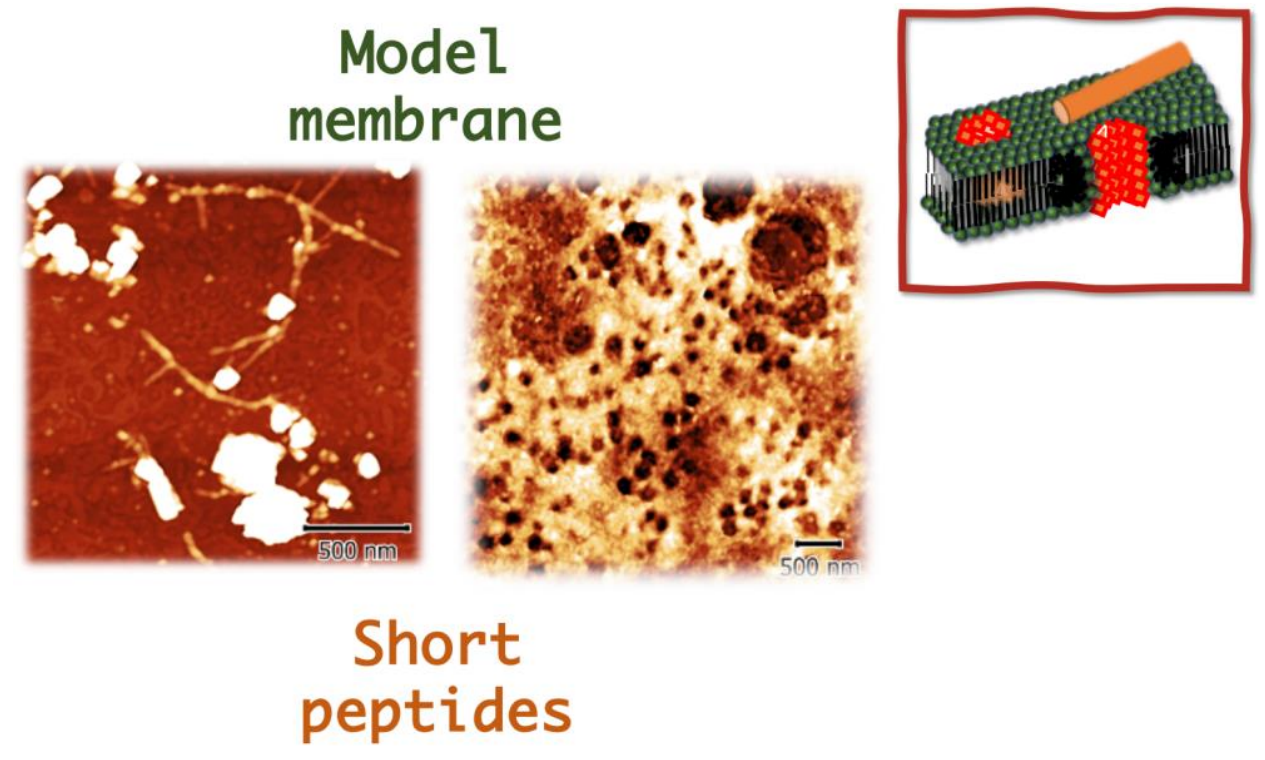

